\title{
COMPUTATIONAL MODELING OF A DYNAMIC KNEE SIMULATOR
}

\author{
S. De Coninck ${ }^{1}$, J. Smis ${ }^{1}$, J. Victor ${ }^{2}$, P. De Baets ${ }^{3}$ and M.A. Verstraete ${ }^{2}$, \\ ${ }^{1}$ Ghent University, Belgium \\ ${ }^{2}$ Ghent University, Department of Physical Medicine and Orthopaedic Surgery, Belgium \\ ${ }^{3}$ Ghent University, Soete Laboratory, Belgium
}

\begin{abstract}
Knee replacement surgery has made a huge evolution during the past decades. For further improvements of knee arthroplasty, dynamic knee simulators are used for fully instrumented ex-vivo tests. This paper provides a closer look at the validation of the rig. Therefore, a hinge model is constructed, resulting in an unambiguous evaluating method. The actual position of the ankle and the reaction forces at the ankle are measured and compared with the simulations. The results indicate an excellent agreement between the outcome of the numerical simulations and the measured values. The kinematics and kinetics of the rig are accordingly considered as validated. In the future, the behaviour of a more realistic knee representation will be evaluated.
\end{abstract}

Keywords: Dynamic Knee Simulator; modeling; kinematics; kinetics

\section{INTRODUCTION}

The first and most widely applied type of knee simulator is the Oxford Knee Rig. This test rig was developed to simulate different flexed-knee stances on cadaveric knees. O'Connor and his team build the first version of the Oxford Rig [1]. The idea of the Oxford Rig was to have a kinematic freedom in the knee. As a consequence, the natural six degrees of freedom (DOF) [2] are allowed to the knee specimen. These DOF describe the three rotations (flexion - extension, varus - valgus and internal - external tibial rotation) and the three translations (anterior - posterior, medial - lateral and proximal - distal). Figure 1 visualizes these DOF. Zavatsky [3] has proved that the Oxford knee rig allows full six DOF of movement to a knee specimen. Dedicated ankle and hip joints are used to facilitate these DOF.

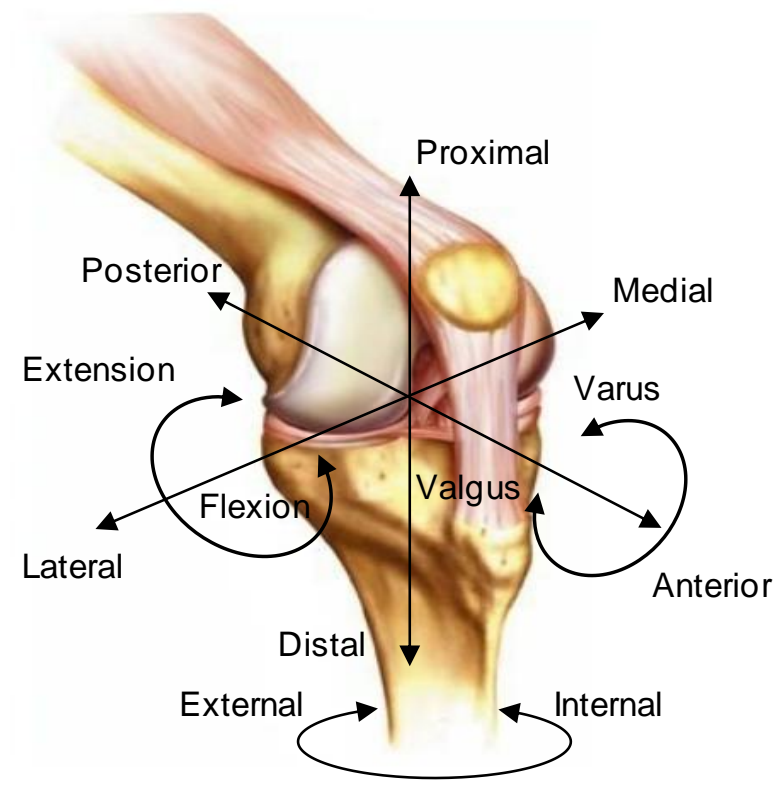

Figure 1: 6 DOF in the knee based on [4]

The Kansas knee simulator [5,6] and the Purdue simulator [7] are based on the Oxford Knee Rig. Different flexion angles could be simulated while keeping the ankle positioned at the vertical axis through the hip. This constraint is partially surmounted by the Enduratec [8] setup, which allows to move the ankle in a small square under the hip position. A limited setup is developed at the New York University for testing high flexion angles. The New York knee simulator [9] has a testing range from $135^{\circ}$ to $155^{\circ}$. Daily movements like walking, cycling, etc. could however not be simulated with the above mentioned setups; a combination of previous rigs is required to test from full extension to high squad angles. In addition, the loads of the 
mentioned rigs are limited to the quadriceps load and the human body weight only. For testing more realistic conditions, there is a need to implement and activate additional muscles, e.g. the hamstrings [10]. On the other hand, the advantage of the setups based on the Oxford Knee Rig is the kinematic freedom they provide to the specimen.

The use of a robot arm overcomes these issues $[11,12]$. In this case, the hip is fixed and the distal tibia is rigidly attached to a robot arm, controlling all six DOF. Next to the quadriceps muscle, an extra pulley system is used, representing the hamstring muscles. A universal force-moment sensor measures the forces between the robot arm and the connection with the tibia. Depending on a specific flexion extension angle, the robot has the purpose to find the position with the lowest reaction forces. This specific position is seen as the static equilibrium of the knee joint. The robot arm is self-learning as the path of the joint is memorized. Subsequently, this path can be repeated with high accuracy after surgical intervention for measuring the influence of the removed ligaments or soft tissues. In this setup, all DOF are controlled by the robot arm. Therefore, the robot arm uses two advanced control algorithms. The first algorithm, a force control mode, uses an iterative procedure to find the equilibrium position of the knee joint. The second algorithm, a position control mode, regenerates the equilibrium path after surgical intervention. The disadvantage of the robot arm is the need of complex algorithms for controlling the six DOF.

To overcome the shortcomings of a traditional Oxford Knee Rig without the need for complicated control algorithms, the UGent Knee Simulator has been defined (see paragraph 2.1). This dynamic knee simulator could be used for several specimens such as cadaveric knees, sawbones and other knee representations. However, without a correct validation, the simulations of these specimens are worthless. In the second paragraph a detailed description of the simulator is provided and the validation models are explained. The result, shown in the third section is leading to the conclusion listed in paragraph 4.

\section{MODEL AND METHODS}

\subsection{UGent Knee Simulator}

The dynamic simulator at Ghent University is built in 2014. Figure 2 gives a sketch of the concept. The knee specimen is left with five DOF. The sixth DOF, the flexion-extension angle, is used as testing parameter. While performing a dynamic simulation, e.g. a cyclic or squatting motion, the position of the ankle is uniquely determined by the flexion extension angle. This sixth DOF then describes the motion and the other five DOF are facilitated by the hip and ankle joint. The hip joint is a hinge around the z-axis. The ankle joint has all rotational DOF combined with an unrestrained translation over the z-axis. This complex joint is mechanically build up by two rotational bearings around different axis. Together with the third combined bearing (rotation and translation), four DOF are given at the ankle. The intersection point of the three rotation axis is located at the center of the translation axis. This specific point theoretically represents the center of the ankle joint.

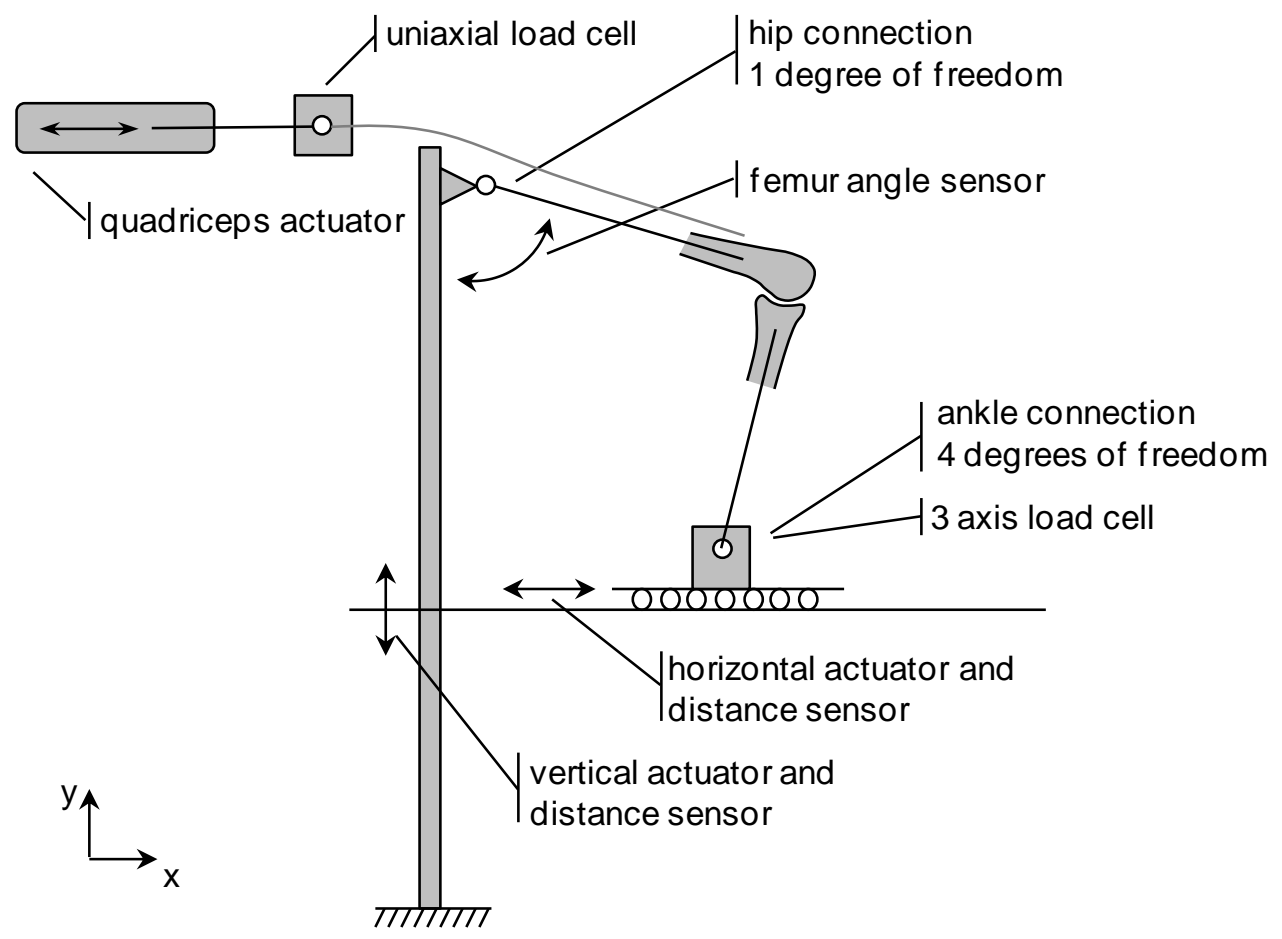

Figure 2: Schematic representation of the knee rig. 
In contrast to an Oxford Knee Rig, the hip position is fixed and the ankle is movable. The ankle position is controlled in the xy-plane of the rig, representing the sagittal plane. The movable ankle, which is driven by a horizontal and vertical actuator, allows simulating realistic human motion like squatting, cycling and gait. This gives the opportunity to test a large range of dynamic simulations. The control unit is written in LabVIEW and uses a synchronized PID controller for the two linear position actuators, one actuator for the $\mathrm{x}$-direction and one for the $\mathrm{y}$-direction. The positions are read out with linear position sensors that are positioned parallel to the linear actuator sleds and used in the feedback loop of the PID control system.

Another important component, next to the ankle build up and the ankle position control, is the quadriceps muscle. This muscle is represented by a linear actuator and is also controlled by a PID controller in the LabVIEW program. The force is applied by a steel cable, guided by a pulley system and attaching at the quadriceps tendon for cadaveric tests. At the linear piston, a uniaxial load cell is attached. This load cell measures the quadriceps forces. At the moment, a similar system is under development for the hamstring muscles.

A second load cell is built between the ankle and the ankle control system. This AMTI load cell measures the reaction forces of the ankle in the three directions. Another measurement tool is the angle sensor. The sensor is fixed at the femur and measures the flexion angle.

The controlling program has two operating modes. In both operating modes, the kinematics (ankle position) and kinetics (applied quadriceps force) are fully disconnected. The difference between the modes is the force control of the rig. The first operating mode is based on the Oxford rig mode. In the Oxford rig, the different flexion angles are achieved by changing the quadriceps forces under a constant body weight and including a movable hip. The UGent Knee Simulator determines the quadriceps force during the movement such that there is a constant ankle reaction force in the vertical y-direction. This constant reaction force, implemented at the ankle, represents the sum of the limb and body weight. In function of the applied ankle motion the knee simulator determines which quadriceps force is required. The same conditions as in the Oxford rig are achieved during this operating mode. The UGent Knee Simulator has also a second operation mode. Instead of implementing a constant reaction force at the ankle, a predefined quadriceps force is now applied without impacting on the kinematics of the ankle joint relative to the hip. The exact load pattern is calculated from musculoskeletal simulations or comes from literature observations. This gives the opportunity to analyze the outcomes of the knee kinetics under a specified quadriceps force and flexion angle.

\subsection{Validation model}

The UGent Knee Simulator is recently built and before executing in vitro experiments a validation is necessary. This validation is needed for detecting building errors and evaluating the control unit. An unambiguous, simplified representation of a knee is used as a validation tool. Such a theoretical model can confirm the correctness of the (force and position) measurements of the UGent Knee Simulator. The model used to describe the knee is a mechanical hinge, as shown in figure 3 . It is a rigid construction were the hinge is represented by bearings mounted on an axis. The beams represent the femur and tibia and the hinge represents the knee joint. Due to the absence of the patella, the quadriceps force is directly applied to the tibia beam. Representing the knee as a hinge reduces the analysis to a two dimensional model. The hinge model is a two-linkage system, with two degrees of freedom. Both DOF are controlled by setting the ankle position. In that case, the kinematics is uniquely defined and independent of the applied forces.

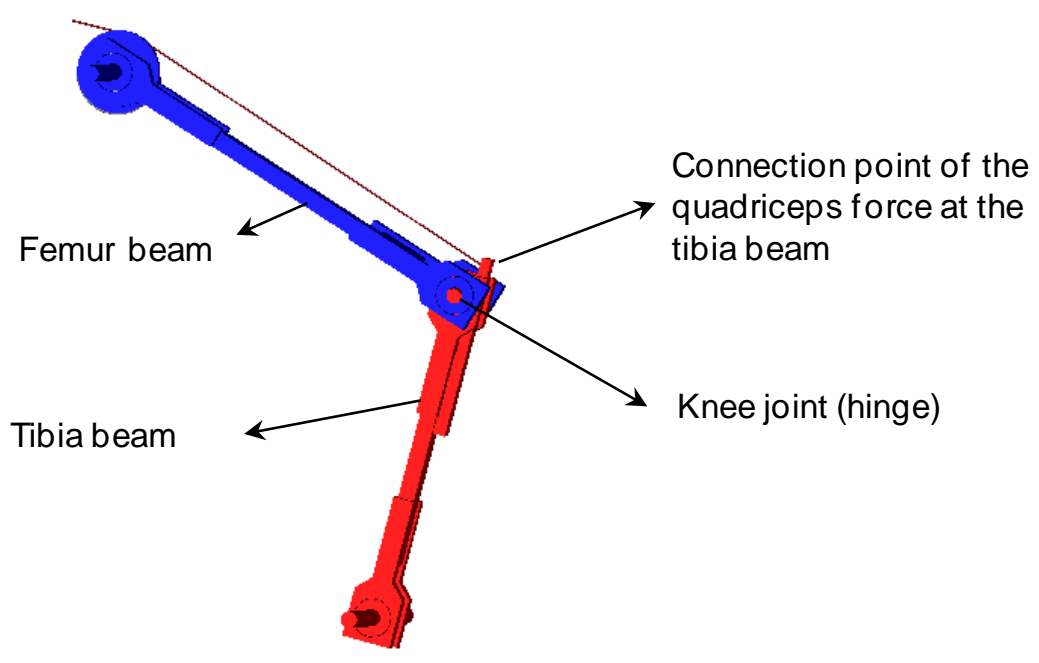

Figure 3: Schematic representation of the mechanical hinge model. 


\subsection{Numerical model}

Matlab is used for modeling this mechanical hinge model. The written program does not only contain kinematics, but also the kinetics of the model for analyzing the ankle reaction forces. To have a realistic model, the inertia of the beams are also included. In the third paragraph, numerical results of the femur angle (Eq. 1-2) and the ankle reaction force (Eq. 3-4) are compared with the measured values obtained from the knee simulator.

$$
\begin{aligned}
& \begin{array}{l}
\text { Angle Difference }=\mid \text { Angle }_{\text {Knee Rig }}-\text { Angle }_{\text {Model }} \mid \\
\text { Relative Angle Difference }=
\end{array}\left|\frac{\text { Angle }_{\text {Knee Rig }} \text { Angle }_{\text {Model }}}{\text { Angle }_{\text {Knee Rig }}}\right| \\
& \text { Force Difference }=\quad\left|F_{\text {Knee Rig }}-F_{\text {Model }}\right| \\
& \text { Relative Force Difference }=\left|\frac{F_{\text {Knee Rig }}-F_{\text {Model }}}{F_{\text {Knee Rig }}}\right|
\end{aligned}
$$

The total reaction force (5) is the vector sum of the force components:

$$
F_{t o t}=\sqrt{F_{x}^{2}+F_{y}^{2}}
$$

The force difference of the quadriceps force compared with the target line is obtained as:

$$
\begin{aligned}
& \text { Quadriceps Force Difference }=\left|F_{q}-F_{q}^{T}\right| \\
& \text { Relative Quadriceps Force Difference }=\left|\frac{F_{q}-F_{q}^{T}}{F_{q}}\right|
\end{aligned}
$$

\subsection{Multi-dynamic model}

The described model in paragraph 2.2 is a simplified knee representation. For more complex knee models, it is easier to use a multi- dynamic program rather than Matlab routines. Several multi dynamic programming tools are used in research. The AnyBody Modelling System (AMS) is a promising tool for biomechanical applications $[13,14]$.

By building up a human body with individual segments, the human body can be analyzed as a mechanical system. The two methods for solving such a system are inverse dynamics and forward dynamics. The AnyBody Modeling System is based on an inverse dynamics approach [15]. Inverse dynamics implies that the internal muscle forces are calculated from the externally applied forces and motion. The external loads on the body and the motions should therefore be known as input. Muscles forces, joint moments, internal (prostheses) positions and (joint reaction) forces are the outputs of the system.

The mechanical hinge model is modeled both in Matlab and in the AnyBody Modeling System. The model of this simplified knee representation will be the basis for future knee models. The advantage of the AMS is the programmed structure of biomechanical objects like bones, muscles, etc... For more complex anatomical and prosthetic joints, the AMS contains a method called Force Dependent Kinematics (FDK) [16]. FDK computes the muscle and reaction forces as well as not-predetermined motion. Modeling a complex knee joint in the AMS is therefore more advantageous than using Matlab routines.

\section{RESULTS}

The test for evaluating the difference between the modeled hinge model and the actual hinge knee is executed using the second working mode of the UGent Knee Simulator. The ankle follows a circle pattern with a radius of $175 \mathrm{~mm}$ and the quadriceps forces is applied as a sinusoidal motion with an amplitude of $100 \mathrm{~N}$ and a mean value of $400 \mathrm{~N}$. The measurements are executed for two cycles and the modeled values are based on the exact ankle position and the exact quadriceps force. The modeled results are those of the program written in Matlab. The modeled results of the multi-dynamic model exactly match the Matlab results. 


\subsection{Kinematics}

The applied ankle motion by the Knee Simulator is shown in figure 4. The offset from the target line is in 95 percent of the measurements smaller than $1.5 \mathrm{~mm}$. In normalized terms, this represents an error lower than $0.86 \%$. The angle sensor of the UGent Knee Simulator is used to analyze the motion of the tested specimen. For the mechanical hinge model, the movement of the beams is uniquely determined by the ankle position. The measured angle values can be verified if the distance between the hip joint and ankle joint is correctly defined. The coordinate transformation between numerical models and the coordinate system of the rig is hereby evaluated. Figure 5 shows the measured and simulated femur angle as function of the ankle position. In absolute terms, the difference in ankle position (Eq. 1) is in 95 percent of the measurements lower than $0.60^{\circ}$. In relative terms, Eq. 2, this represents an error lower than $1.5 \%$.

(a)

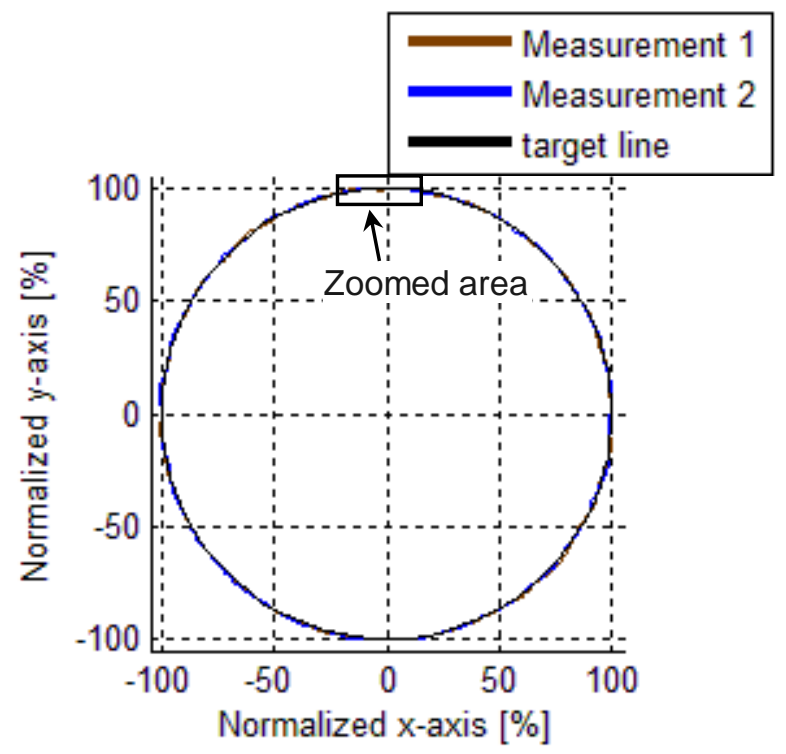

(b)

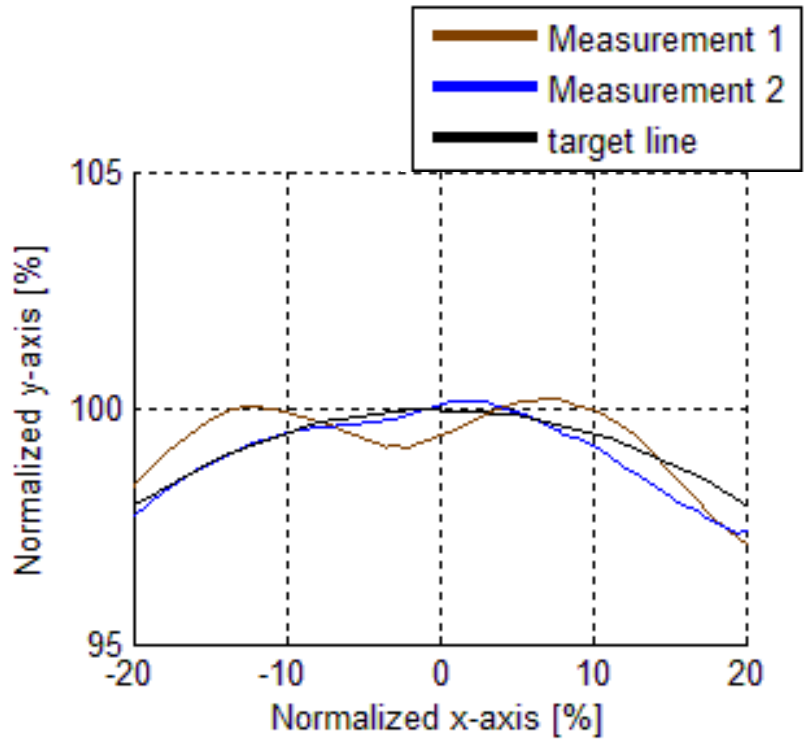

Figure 4: The applied ankle motion of the mechanical hinge by the Knee Simulator with the center point in the middle and normalized to the wanted circle radius $(175 \mathrm{~mm})$. The entire cycle loop is demonstrated in (a) and a zoomed selection in (b).

(a)

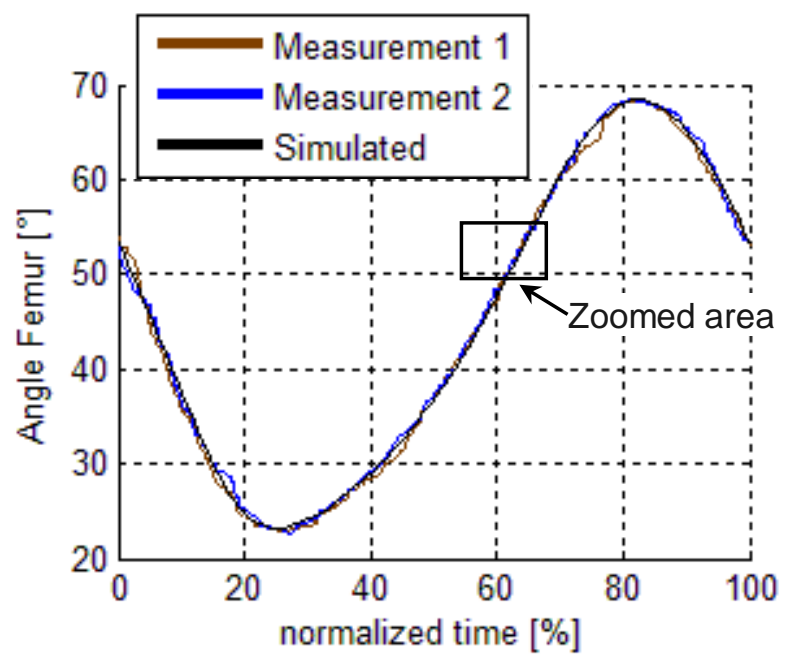

(b)

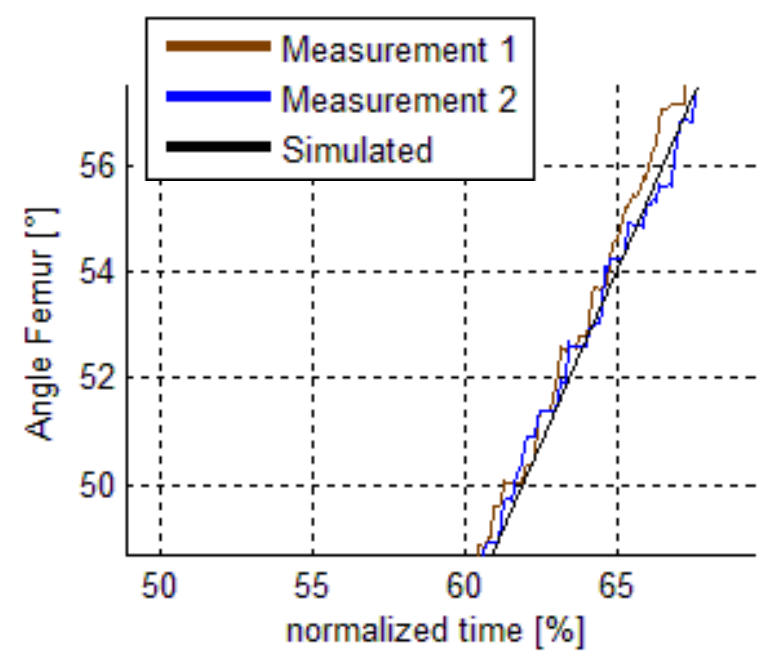

Figure 5: The measured femur angles and the simulated femur angle during a circle motion. The entire cycle loop is demonstrated in (a) and a zoomed selection in (b) 


\subsection{Kinetics}

The measured reaction forces at the ankle load cell are compared with the numerically simulated values. Figure 6.a shows the total reaction force (Eq. 5) of two measurements during a cyclic motion. The variance of the measurements is mainly influenced by the variance of the applied quadriceps force which is shown in Figure 6.c. This figure indicates that the applied quadriceps force follows the target line relatively well, with an error (Eq. 6) lower than $16.4 \mathrm{~N}$ in 95 percent of the measurements. In relative terms, Eq. 7, this represent an error lower than $4.4 \%$. Figure $6 . \mathrm{b}$ compares the forced measured during the first cycle with a range of simulated values. This simulated range comes from the margin at the tibial attachment point of the quadriceps muscle. This attachment point has a margin of $2.5 \mathrm{~mm}$, which obviously influences the total ankle reaction force. The attachment point is an unstable point between these margins. Therefore, the attachment of the quadriceps force varies between extremes. The maximum and minimum values of the simulated range plotted in figure 6.b correspond to the extremes of this margin. Accordingly, the force difference (Eq. 3 ) is described as the minimum difference with either of these two extreme positions.

Figure 6.b shows a relative high force difference around $65 \%$ of the normalized cycle time. At that moment the total externally applied force (quadriceps force and gravitational force) reaches its highest magnitude while the reaction force at the ankle is at its lowest. This represents an extremely sensitive situation for the system. In general, however, the measured values including these force errors match very well with the numerical ones. The force difference (Eq. 3) is lower than $3.5 \mathrm{~N}$ for $95 \%$ of the measured values, representing a relative force difference (Eq. 4) lower than $3.6 \%$.

(a)

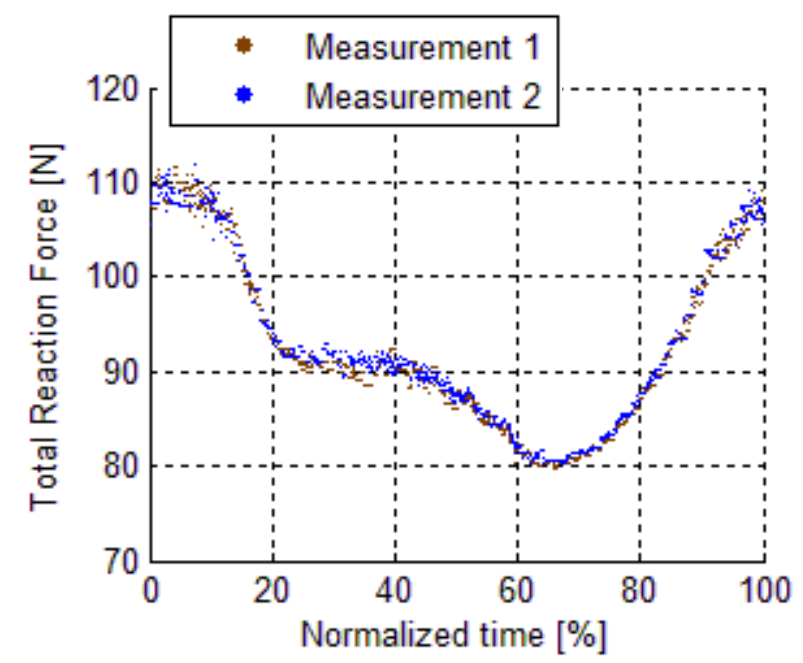

(b)

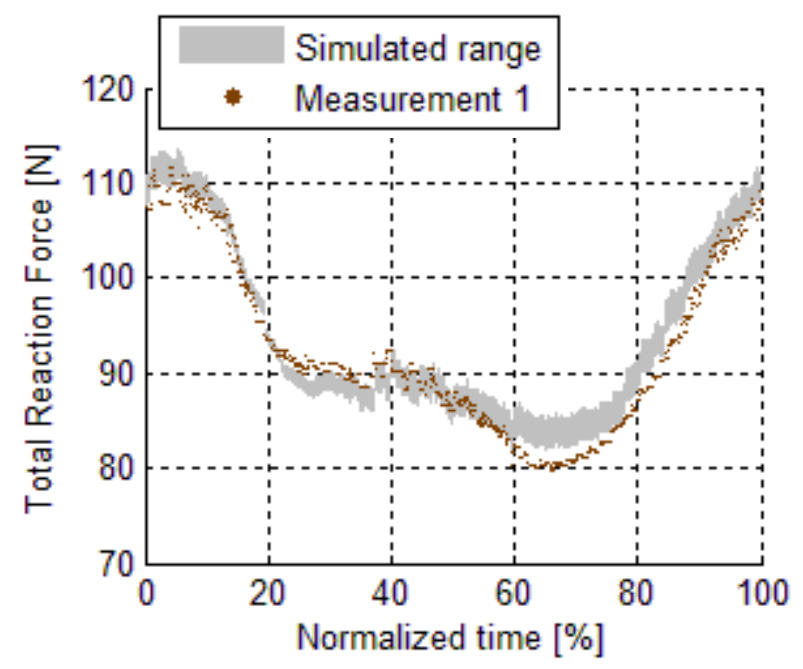

(c)

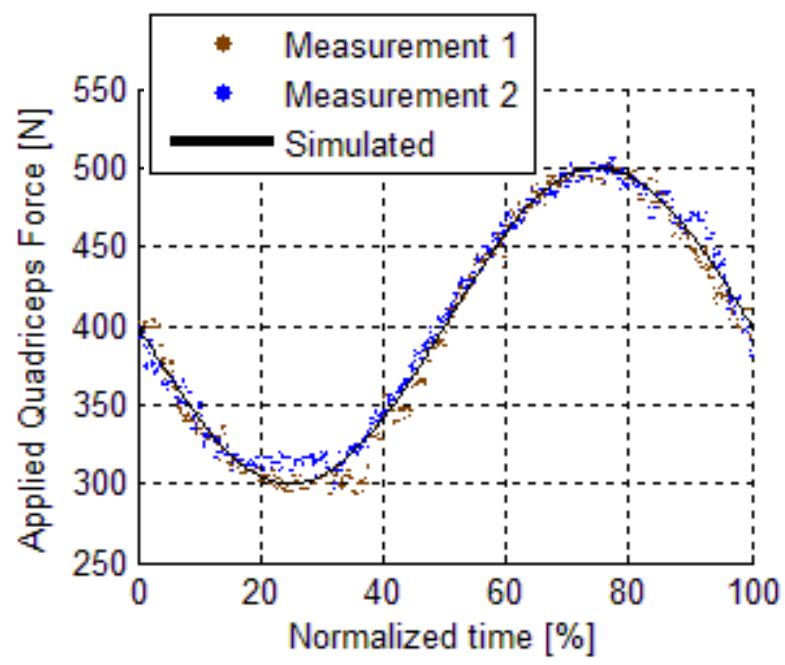

Figure 6: Modelling and measuring of the hinge model at the UGent Knee Simulator. a) The total ankle reaction force of the two measurements, b) Measurement 1 of the ankle force compared with the simulated surface ,c) The applied quadriceps force of the measurements. 


\section{CONCLUSIONS}

A new knee simulator for testing cadaveric knee specimens has recently been developed. The UGent Knee Simulator combines the advantages of existing knee rigs. The rig will be used for evaluating dynamic simulations representing activities of daily living such as cycling, squatting or gait movements. A validation with a two dimensional model indicates very accurate control loops. Based on a mechanical hinge model, simplifying a knee, the kinematics and kinetics have been evaluated. The kinematics are exactly defined by the UGent Knee Simulator without any influence of the applied forces. The kinematic evaluation results in an accuracy of $1.5 \mathrm{~mm}$ for the dynamic control of the Knee Simulator. For measuring actual positions an angle sensor is used. The absolute difference in the femur angle is in 95 percent of the measurements lower than $0.60^{\circ}$. In relative terms, this represents an error lower than $1.5 \%$. The kinetics encompass the evaluation of the ankle reaction forces and quadriceps force. The force difference between the measured ankle reaction forces and modelled forces is lower than $3.5 \mathrm{~N}$ for $95 \%$ of the measured values. This results in a relative force difference lower than $3.6 \%$. The measured results (kinematics and kinetics) are very accurate. The validation gives us a correct working dynamic simulator, ready for executing medical research tests. This simplified model will be used in the design of a three dimensional Total Knee Replacement (TKR).

\section{NOMENCLATURE}

\begin{tabular}{|c|c|c|}
\hline$x, y, z$ & \multicolumn{2}{|c|}{ Axis of the right handed Cartesian coordinate system } \\
\hline DOF & \multicolumn{2}{|l|}{ Degrees Of Freedom. } \\
\hline PID & \multicolumn{2}{|l|}{ Proportional-integral-derivative } \\
\hline AMS & \multicolumn{2}{|l|}{ AnyBody Modelling System } \\
\hline FDK & \multicolumn{2}{|l|}{ Force Dependent Kinematics } \\
\hline OptiTrack & \multicolumn{2}{|l|}{ Motion capture system } \\
\hline TKR & \multicolumn{2}{|l|}{ Total Knee Replacement } \\
\hline $\mathrm{F}$ & Force & $\mathrm{N}$ \\
\hline$F_{t o t}$ & Total reaction force & $\mathrm{N}$ \\
\hline$F_{x}$ & Force in the $\mathrm{x}$ direction & $\mathrm{N}$ \\
\hline$F_{y}$ & Force in the $\mathrm{x}$ direction & $\mathrm{N}$ \\
\hline$F_{q}$ & Measured quadriceps force & $\mathrm{N}$ \\
\hline \multirow[t]{3}{*}{$F_{q}^{T}$} & Target line of the quadriceps force & $\mathrm{N}$ \\
\hline & Degrees & $\circ$ \\
\hline & Length & $\mathrm{mm}$ \\
\hline
\end{tabular}

\section{ACKNOWLEDGEMENTS}

\section{REFERENCES}

[1] J. W. Bourne R., Goodfellow and O'Connor J.J., A functional analysis of various knee arthroplasties, trans. orthop. res., 24-160, 1978.

[2] Bull, A. M. J., and A. A. Amis. "Knee joint motion: description and measurement." Proceedings of the Institution of Mechanical Engineers, Part H: Journal of Engineering in Medicine 212.5 (1998): 357-372.

[3] $A B$ Zavatsky. A kinematic-freedom analysis of a flexed-knee-stance testing rig. Journal of biomechanics, 30(3):277-280, 1997.

[4] http://www.greatrivermedical.org/orthopedics/faqs/partial-knee.php

[5] Trent $M$ Guess and Lorin P Maletsky. Computational modeling of a dynamic knee simulator for reproduction of knee loading. Journal of biomechanical engineering, 127(7):1216-1221, 2005. 
[6] Halloran, Jason P., et al. Verification of predicted knee replacement kinematics during simulated gait in the Kansas knee simulator. Journal of biomechanical engineering 132.8 (2010): 081010.

[7] Lorin P Maletsky and Ben M Hillberry. Simulating dynamic activities using a five-axis knee simulator.Journal of biomechanical engineering, 127(1):123-133, 2005.

[8] JL Pavlovic, SJ Kirstukas, H Touchi, and JE Bechtold. Dynamic simulation machine for measurement of knee mechanics and intra-articular pressures. ASME-PUBLICATIONS-BED, 28:277-277, 1994.

[9] Gokce Yildirim, Peter S Walker, Jon Sussman-Fort, Gaurav Aggarwal, Brian White, and Gregg R Klein. The contact locations in the knee during high flexion. The Knee, 14(5):379-384, 2007.

[10] G Li, TW Rudy, M Sakane, A Kanamori, CB Ma, and SL-Y Woo. The importance of quadriceps and hamstring muscle loading on knee kinematics and in-situ forces in the acl.Journal of biomechanics, 32(4):395-400, 1999.

[11] TW Rudy, GA Livesay, SL-Y Woo, and FH Fu. A combined robotic/universal force sensor approach to determine in situ forces of knee ligaments. Journal of biomechanics, 29(10):1357-1360, 1996.

[12] G Li, LE DeFrate, S Zayontz, SE Park, and TJ Gill. The effect of tibiofemoral joint kinematics on patellofemoral contact pressures under simulated muscle loads. Journal of orthopaedic research, 22(4):801-806, 2004.

[13] Damsgaard, Michael, et al. Analysis of musculoskeletal systems in the AnyBody Modeling System. Simulation Modelling Practice and Theory 14.8 (2006): 1100-1111.

[14] Bonin, Dominik, et al. Exchanging data between Digital Human Modelling systems: a review of data formats. (2014).

[15] Rasmussen, John, et al. Anybody-a software system for ergonomic optimization. Fifth World Congress on Structural and Multidisciplinary Optimization. Vol. 4. 2003.

[16] Andersen, Michael Skipper, Michael Damsgaard, and John Rasmussen. "Force-dependent kinematics: a new analysis method for non-conforming joints." 13th Biennial International Symposium on Computer Simulation in Biomechanics. 2011. 